\title{
Transient Responses of Saccharomyces uvarum to a Change of the Growth-limiting Nutrient in Continuous Culture
}

\author{
By OTHMAR K ̈̈PPELI, * MAGDALENA GSCHWEND-PETRIK AND \\ ARMIN FIECHTER \\ Department of Biotechnology, Swiss Federal Institute of Technology, Hoenggerberg, \\ $\mathrm{CH}-8093$ Zürich, Switzerland
}

(Received 1 June 1984; revised 14 August 1984)

\begin{abstract}
The transition of Saccharomyces uvarum from oxidative to oxido-reductive glucose metabolism was characterized by the immediate formation of ethanol and the gradual adaptation of cells to a new physiological state, as expressed by a decrease in mitochondrial cytochrome content and a loss of malate dehydrogenase activity. Shifting the cells from carbon-limited to oxygen-, nitrogen- and iron-limited media in a continuous culture growing oxidatively led to the initiation of the same effects. The results lend support to the concept of a limited respiratory capacity as the basis for the occurrence of oxido-reductive glucose metabolism. Limitation of growth by a nutrient other than glucose results in an imbalance between glucose flux and biosynthetic potential of the cells and causes oxido-reductive glucose breakdown.
\end{abstract}

\section{INTRODUCTION}

The bulk of our existing knowledge of transient phenomena, such as enzyme induction and repression, originates from observations on batch cultures with the carbon and energy source at supersaturating concentrations. However, in most natural environments subsaturating concentrations of the energy source are usual (Tempest \& Neijssel, 1976). For the maintenance of microbial cultures under nutrient-limited growth conditions, continuous culture is necessary. This technique has been widely used for studies on microbial physiology at steady state conditions (Tempest \& Neijssel, 1976). However, little information is available about the responses of microbial cell cultures to changes in, for example, the limiting nutrient(s) or the growth rate. Furthermore, existing information about the transient behaviour of microbial cultures mainly concerns bacteria (Ryu \& Mateles, 1968; Dean, 1972; Ashby \& Harrison, 1980).

The recording of transient responses of a yeast culture growing continuously yields insight into regulatory processes. Galindez et al. (1983) investigated the induction and repression of the galactose pathway enzymes by changing the medium feed from glucose to glucose and galactose in induction experiments and from galactose to galactose and glucose in repression experiments.

We have studied the aerobic glucose metabolism of Saccharomyces cerevisiae and Saccharomyces uvarum extensively by means of the continuous culture technique. Steady-state analysis, glucose pulse and dilution rate shift experiments have revealed that the so-called 'glucose effect' is based primarily on the limited capacity of the oxidative pathways (Rieger $e t$ al., 1983). This leads to an alternative decarboxylation of pyruvate, with ethanol as the end product, when the glycolytic flux is beyond the oxidative capacity. Since part of the glucose is still oxidatively utilized, glucose breakdown with the concomitant formation of ethanol is related to an oxido-reductive glucose metabolism (Rieger et al., 1983). When the cells are kept in conditions of oxido-reductive glucose metabolism for 24 to $48 \mathrm{~h}$, an adaptation occurs, as manifested by a decrease in mitochondrial cytochrome content (Petrik et al., 1983). These investigations show that ethanol formation is not the consequence of the repression of oxidative pathways, but results from an exhaustion of pyruvate oxidation capacity. 
Since the concept of a limited oxidative capacity of the cells corresponds to the appearance of a limitation within the cell, the response of the cells to externally introduced nutrient limitations is of interest. We report here on the transient responses of various parameters (overall metabolism, cytochrome content, and malate dehydrogenase activity) of $S$. uvarum to oxygen, nitrogen and iron limitation. The basic response pattern of these parameters to a transition from oxidative to oxido-reductive glucose metabolism was determined by measurement after a shift in dilution rate.

\section{METHODS}

Organism. Saccharomyces utarum $\mathrm{H} 2055$ (from this department) was used in all the experiments.

Media. A chemically defined medium, with the following composition per mole of carbon, was used throughout this work: $\left(\mathrm{NH}_{4}\right)_{2} \mathrm{SO}_{+}, 6 \mathrm{~g}:\left(\mathrm{NH}_{4}\right)_{2} \mathrm{HPO}_{4}, 1.95 \mathrm{~g}: \mathrm{KCl}, 0.9 \mathrm{~g}: \mathrm{MgSO}_{4} .7 \mathrm{H}_{2} \mathrm{O}, 0.45 \mathrm{~g}: \mathrm{CaCl}_{2} .2 \mathrm{H}_{2} \mathrm{O}, 0.3 \mathrm{~g}$; $\mathrm{CuSO}_{+} \cdot 5 \mathrm{H}_{2} \mathrm{O}, 2.34 \mathrm{mg}: \mathrm{FeCl}_{3} \cdot 6 \mathrm{H}_{2} \mathrm{O}, 14.4 \mathrm{mg}: \mathrm{ZnSO}_{+} \cdot 7 \mathrm{H}_{2} \mathrm{O}, 9 \mathrm{mg} ; \mathrm{MnSO}_{4} .2 \mathrm{H}_{2} \mathrm{O}, 10.5 \mathrm{mg}$; biotin, $0.03 \mathrm{mg}$; mirr-inositol. $60 \mathrm{mg}$ : calcium pantothenate. $30 \mathrm{mg}$; thiamin hydrochloride, $6 \mathrm{mg}$ : pyridoxine hydrochloride, $1.5 \mathrm{mg}$.

In its original composition, this medium has a $\mathrm{C} / \mathrm{N}$ ratio of 7.7 and is carbon-limited. In the experiments described, the glucose concentration was $55 \mathrm{~g}^{-1}$. The medium was heat-sterilized $\left(12 \mathrm{I}^{\circ} \mathrm{C}, 20 \mathrm{~min}\right)$ after reducing the $\mathrm{pH}$ to $2 \cdot 5-3 \cdot 0$ with concentrated hydrochloric acid. For continuous cultivation, sterilization was carried out in 300 litre batches.

Nitrogen-limited medium was prepared by omitting $\left(\mathrm{NH}_{4}\right)_{2} \mathrm{SO}_{4}$, the only nitrogen source besides $\left(\mathrm{NH}_{4}\right)_{2} \mathrm{HPO}_{4}$, from the original medium. This increased the $\mathrm{C} / \mathrm{N}$ ratio to 30 . Sulphur limitation was prevented by using sulphuric acid to acidify the nitrogen-limited medium.

Iron-limited medium was obtained when iron was completely omitted from the medium. In this medium, an iron concentration of $0.5 \mathrm{mg} \mathrm{l}^{-1}$ was measured. This amount originated from the stainless steel vessels and contamination of other medium salts.

For oxygen-limited growth, the inlet air was replaced by an oxygen/nitrogen mixture which contained $2.4 \%$ $(\mathrm{v} / \mathrm{v})$ oxygen. The oxygen partial pressure of the culture liquid dropped to 0 when this mixture was used.

Culture conditions. The organisms were grown in a 10 litre bioreactor (Chemap AG, Männedorf, Switzerland) equipped with a flat-blade turbine stirrer. The working volume was kept constant at 3 litres by a syphon. A pH of 5.5 was maintained automatically with $10 \mathrm{~mol} \mathrm{NaOH} \mathrm{l}^{-1}$. The reactor was aerated with air at a flow rate of 300 $480 \mathrm{l} \mathrm{h}^{-1}$, corresponding to 1.7 .2 .7 vols air per vol. liquid $\mathrm{min}^{-1}$. These aeration rates yielded oxygen partial pressures above $30 \%$ of air saturation. The temperature was constant at $30^{\circ} \mathrm{C}$. For agitation, a stirring speed of 900 r.p.m. was used. For a constant medium flow during continuous cultivation, Watson-Marlow MHRE 100 flow inducers (Falmouth, UK) were used.

Shift technique. Shifts in dilution rate were achieved by increasing the flow rate of the inflowing medium. Oxygen shifts were made by changing the inlet air to the above-mentioned oxygen/nitrogen mixture. In all cases, the carbon-limited medium was the starting point: for shifts to nitrogen or iron limitation. it was replaced by the appropriate nutrient-limited medium.

Sampling. Samples were withdrawn with a syringe, the needle being inserted into the reactor through a membrane.

Determination of biomass, glucose, ethanol and acetate. For measuring biomass concentration, $10 \mathrm{ml}$ culture liquid was centrifuged at $2000 \mathrm{~g}$ for $5 \mathrm{~min}$ in tared centrifuge tubes. The sediment was washed twice with distilled water and then dried to constant weight at $105^{\circ} \mathrm{C}$.

Glucose was determined in a glucose analyser (YSI, Yellow Springs, Ohio, USA) with a detection limit of $100 \mathrm{mg} \mathrm{l}^{-1}$. Ethanol and acetate were analysed by gas chromatography (Hewlett Packard gas chromatograph $5830 \mathrm{~A}$, Avondale, $\mathrm{Pa}$., USA). Prior to injection, the cells were removed by centrifugation and known amounts of methanol and propionic acid were added as internal standards. The columns were as follows: for ethanol. Porapack QS. mesh $100 \cdot 120$, stainless steel column. $6 \mathrm{ft}(1.8 \mathrm{~m})$ : for acetate. $10^{\circ}{ }_{0}\left(\mathrm{w}\right.$ w) $\mathrm{SP}-1200,1^{\circ}{ }_{0}(\mathrm{w}: \mathrm{w}) \mathrm{H} \mathrm{PO}_{4}$ on 80100 Chromosorb WAW, glass column. $6 \mathrm{ft}(1.8 \mathrm{~m})$. For both columns the temperature was $150 \mathrm{C}$.

Gas exchange rates. The specific oxygen uptake rate $\left(Q_{\omega_{2}}\right)$ and carbon dioxide production rate $\left(Q_{\left(0_{2}\right.}\right)$ were calculated from the data provided by a gas analyser (Hartmann \& Braun, FRG) connected to the air outlet of the reactor, and were based on an inert gas balance.

Determination of cytochrome content. This was done according to Gmünder et al. (1981).

Preparation of cell-free extracts and determination of malate dehydrogenase activity. Cells ( 3 to $5 \mathrm{~g}$ wet $\mathrm{w}$ ) collected from the bioreactor were centrifuged and washed with $100 \mathrm{mM}$-phosphate buffer, $\mathrm{pH} 7.5$. The sediment was resuspended in the same buffer, together with glass beads $(0.0250 .30 \mathrm{~mm})$. The mixture was ground for 40 to $50 \mathrm{~s}$ using a hand-mixer equipped with a notched disk. Unbroken cells and cell debris were removed by centrifugation at $8000 \mathrm{~g}$ for $10 \mathrm{~min}$. 

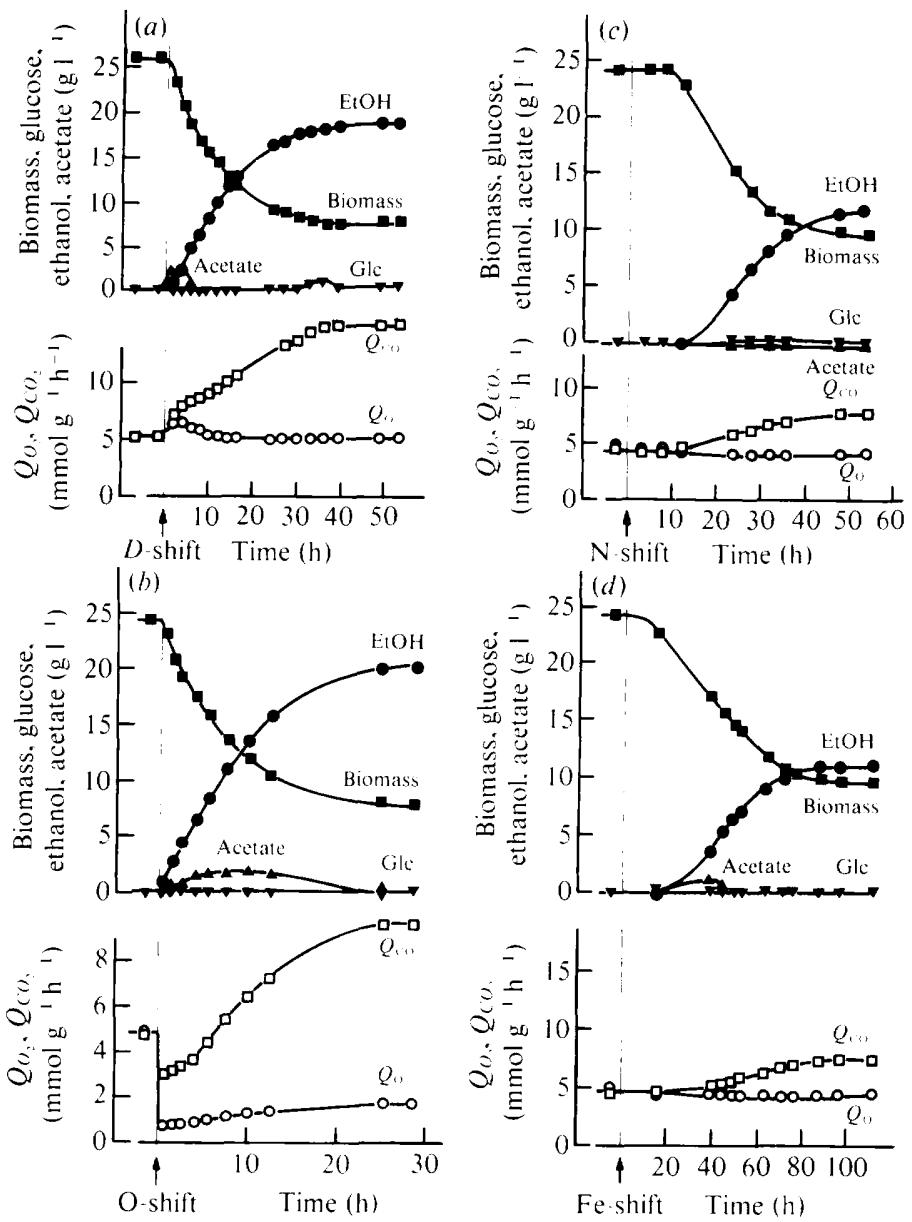

Fig. 1. Transient responses of the overall metabolism of $S$. utarum to changes in cultivation conditions : (a) shift in dilution rate from $0.14 \mathrm{~h}^{-1}$ to $0.21 \mathrm{~h}^{-1}$, i.e. shift from oxidative to oxido-reductive glucose metabolism; (b) shift from carbon to oxygen limitation $\left(D=-0.15 \mathrm{~h}^{-1}\right) ;(c)$ shift from carbon to nitrogen limitation $\left(D=\left(0.13 \mathrm{~h}^{-1}\right)\right.$ : and $(d)$ shift from carbon to iron limitation $\left(D=0 \cdot 14 \mathrm{~h}^{-1}\right)$. $\mathbf{\square}$, Biomass: ethanol: $\boldsymbol{\Lambda}$, acetate: $\boldsymbol{\nabla}$, residual glucose : $\bigcirc, Q_{\mathrm{CO}}: \square, Q_{\mathrm{O}_{2}}$.

Malate dehydrogenase activity was determined according to Flury et al. (1974). One unit of enzyme activity is equivalent to the formation of $1 \mu \mathrm{mol} \mathrm{NAD}{ }^{+} \min ^{-1}$ at $30^{\circ} \mathrm{C}$. Protein was assayed by the Lowry method with bovine serum albumin as the standard.

\section{RESULTS}

In continuous culture, $S$. utarum degrades glucose oxidatively up to a dilution rate of $0 \cdot 16 \mathrm{~h}^{-1}$. At higher dilution rates, the breakdown of glucose is oxido-reductive, with ethanol formation (Petrik et al., 1983). This behaviour is due to the limited respiratory capacity of the cells, which is exhausted at the maximum growth rate for oxidative breakdown of glucose (Rieger et al., 1983).

In order to establish the response of the cells when the maximum growth rate for oxidative glucose metabolism is exceeded, the dilution rate was shifted from $0 \cdot 14 \mathrm{~h}^{-1}$ to $0 \cdot 21 \mathrm{~h}^{-1}$. The response of the overall metabolism to such a shift is shown in Fig. 1(a). The immediate accumulation of ethanol (and some acetate at the beginning of the shift) was paralleled by decreasing biomass concentrations. The gas exchange rates indicated the onset of oxido- 

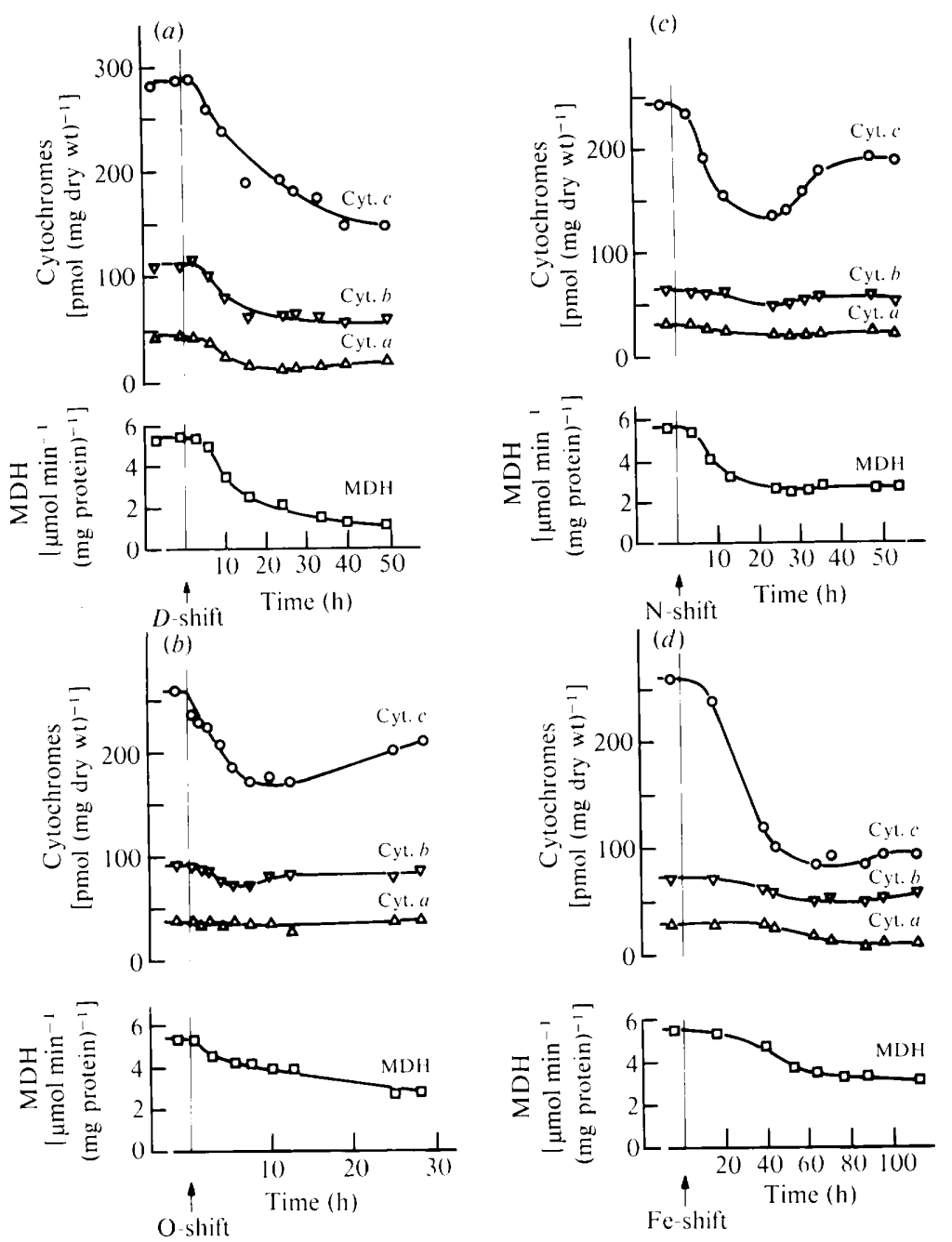

Fig. 2. Transient responses of mitochondrial cytochrome content and malate dehydrogenase (MDH) activity of $S$. uvarum to changes in cultivation conditions: $(a),(b),(c)$ and $(d)$ as in Fig. $1 . \triangle$, Cytochrome $a: \nabla$, cytochrome $h ; O$. cytochrome $c: \square$. malate dehydrogenase specific activity.

reductive glucose metabolism, with an increased carbon dioxide production rate. A gradual decrease in cytochrome content and in the specific activity of malate dehydrogenase also occurred (Fig. $2 a$ ). After approximately $48 \mathrm{~h}$, all parameters reached the steady-state values for oxido-reductive glucose metabolism (Table 1).

\section{Shift from carbon to oxygen limitation}

Oxygen limitation was introduced at the steady state of a culture growing at a dilution rate of $0 \cdot 15 \mathrm{~h}^{-1}$ and consequently exhibiting oxidative glucose metabolism. The culture responded with immediate formation of ethanol and acetate (Fig. $1 b$ ). Biomass concentration decreased. Gas exchange rates dropped and then increased. The increase of the carbon dioxide production rate was more pronounced than that of the oxygen uptake rate. Again, gas exchange rates indicated oxido-reductive glucose metabolism.

Generally, the response of the overall culture parameters very closely resembled that seen after the dilution rate shift. The same was true for the responses in mitochondrial cytochrome 
Table 1. Minimum and maximum values for cytochrome content and specific activity of malate dehydrogenase $(M D H)$ of $S$. uvarum during oxidative and oxido-reductive glucose metabolism in continuous culture

\begin{tabular}{|c|c|c|c|c|}
\hline \multirow[b]{2}{*}{ Glucose metabolism } & \multicolumn{3}{|c|}{$\begin{array}{l}\text { Cytochrome content } \\
{\left[\mathrm{pmol}(\mathrm{mg} \text { dry cell })^{-1}\right]}\end{array}$} & \multirow{2}{*}{$\begin{array}{l}\mathrm{MDH} \\
\text { (units) }\end{array}$} \\
\hline & Cyt. $a$ & Cyt. $b$ & Cyt. $c$ & \\
\hline \multicolumn{5}{|l|}{ Oxidative: } \\
\hline$D=0.05 \mathrm{~h}^{-1}$ & 30 & 60 & 150 & 5 \\
\hline$D=0.15 \mathrm{~h}^{-1}$ & 50 & 100 & 310 & 6 \\
\hline \multicolumn{5}{|l|}{ Oxido-reductive: } \\
\hline$D=0.17 \mathrm{~h}^{-1}$ & 20 & 65 & 170 & 2 \\
\hline$D=0.25 \mathrm{~h}^{-1}$ & 10 & 50 & 120 & 1 \\
\hline
\end{tabular}

content and the specific activity of malate dehydrogenase (Fig. $2 b$ ), although minor differences were noticed in the dynamics, which probably originated from the different dilution rates in the two experiments.

\section{Shift from carbon to nitrogen and iron limitation}

The responses of the cells to the introduction of these two nutrient limitations were very similar (Fig. 1c,d). Because the carbon-limited medium contained a surplus of nitrogen and iron, the limitation did not become effective until the residual nitrogen or iron had washed out of the bioreactor; this took 10 to $15 \mathrm{~h}$. After this time, ethanol and small amounts of acetate started to accumulate in the culture liquid and the biomass concentration decreased accordingly. Again, the gas exchange rates indicated the beginning of oxido-reductive glucose breakdown, with the carbon dioxide production rate higher than the oxygen uptake rate.

As a consequence of the onset of oxido-reductive glucose metabolism, the responses of mitochondrial cytochrome content and malate dehydrogenase activity were analogous to those noticed after the shift in dilution rate and the introduction of oxygen limitation (Fig. $2 c, d$ ). There were some differences in the degree of decrease in cytochrome content and malate dehydrogenase activity, and the dynamics also differed somewhat from the pattern seen with the dilution rate shift. This may have been because the dilution rates again were not identical in the experiments compared. Basically, there was considerable congruence in the responses produced by the different experiments.

\section{DISCUSSION}

The events taking place when $S$. uvarum cells are shifted from oxidative to oxido-reductive glucose metabolism have been described as short- and long-term regulation (Petrik et al., 1983). The short-term regulation is expressed by the occurrence of ethanol immediately after conditions for oxido-reductive metabolism are introduced. This primary regulation corresponds to an overflow reaction, at the level of pyruvate, when the oxidative branch of glucose metabolism is saturated.

The long-term regulation taking place during the transition from oxidative to oxido-reductive glucose metabolism is a gradual adaptation of the cells to this physiological state. It is represented by the analysis of mitochondrial cytochrome content, which exhibits a decrease from high concentrations at oxidative glucose breakdown to low concentrations at oxidoreductive glucose metabolism (Table 1). The same pattern is found for the specific activity of malate dehydrogenase (Table 1).

We have established that oxido-reductive glucose metabolism is the consequence of a limited respiratory capacity, which means that the glycolytic flux is beyond the oxidative capacity of the cells. It occurs when excess glucose is present or when the cells grow at elevated dilution rates (above $0 \cdot 16 \mathrm{~h}^{-1}$ for $S$. uvarum). This concept is equivalent to the occurrence of a limitation within the cell in the oxidative branch of metabolism. The shifting of the cells to oxygen-limited growth represents the artifical introduction of a lower oxidative capacity, as a consequence of 
the lower availability of the terminal electron acceptor of the respiratory chain. The response of the cells to oxygen limitation (Fig. $1 b$, Fig. $2 b$ ) and its similarity to that of the shift from oxidative to oxido-reductive glucose metabolism (Fig. $1 a$, Fig. $2 a$ ) indicates that the supposed basis for the appearance of oxido-reductive glucose metabolism is correct.

It follows that the so-called 'glucose effect' (i.e. initiation of oxido-reductive glucose metabolism by excess glucose) and the Pasteur effect (Pasteur, 1861) are closely related in that the latter is a premature triggering of the 'glucose effect' by oxygen limitation and therefore represents a special case of the 'glucose effect.' This is in accordance with the definition of the Pasteur effect as the inhibition of ethanol formation of oxygen. The transition from carbon- to oxygen-limited growth in continuous culture shows that oxygen effectively exerts this function since glucose metabolism is changed from oxidative to oxido-reductive by the shift.

Our results further showed that other nutrient limitations (iron and nitrogen) have an effect analogous to oxygen limitation (Fig. $1 c, d$, Fig. $2 c, d$ ). With these two nutrient limitations, a change from oxidative to oxido-reductive glucose metabolism is initiated, with kinetics similar to those of the shift in dilution rate (Fig. $1 a$, Fig. $2 a$ ). It follows that not only a limitation of respiration causes a change in glucose metabolism, but any general inhibition of growth which leads to a supercritical flux of glycolysis.

In a carbon-limited continuous culture, the feed rate of glucose is balanced with the actual growth rate, in that growth is limited by the supply of the carbon source. This culture technique allows the lowering of feed rate, and consequently of growth rate, to a level where oxidative glucose breakdown is possible. When the growth-limiting nutrient in a culture oxidatively metabolizing glucose is changed from carbon to any other medium component, the equilibrium between growth and carbon source feed is disturbed. Theoretically, glucose should accumulate in the culture liquid. However, since glucose uptake and glycolysis are not regulated according to the biosynthetic capacity of the cells, oxido-reductive glucose metabolism is initiated. These results show that any limitation of the biosynthetic branch of metabolism will also lead to ethanol formation. It is concluded that a saturated respiratory capacity causes oxido-reductive glucose metabolism at a particular growth rate in an aerobic continuous culture because the maximum growth rate of the cells is higher than that noted for oxidative glucose metabolism $\left(0 \cdot 26 \mathrm{~h}^{-1}\right.$ and $0 \cdot 16 \mathrm{~h}^{-1}$, respectively, for $S$. uvarum: Petrik et al., 1983).

This work was supported by grant 3.051-0.81 of the Swiss National Foundation.

\section{REFERENCES}

Ashby, R. E. \& Harrison, D. E. F. (1980). Studies on the induction and turnover of the nitrate-oxidizing capacity in Klebsiella aerogenes using chemostat culture. Journal of General Microbiology 120, 465473.

DEAN, A. C. R. (1972). Influence of environment on the control of enzyme synthesis. Journal of Applied Chemistry and Biotechnology 22, 245-259.

Flury, U., Heer, B. \& Fiechter, A. (1974). Isoenzyme pattern of malate dehydrogenase during respiratory derepression in Schizosaccharomyces pombe. Archives of Microbiology 97, 141-148.

Galindez, M. J., Ruizordaz, N., Herrero, P. \& Moreno, F. (1983). Transient responses of yeast continuous cultures to qualitative changes in the nutrient supply. Induction and repression of the galactose pathway enzymes. Archives of Microbiology 135, 115-119.

GMÜNDER, F. K., Kä PPELI, O. \& FiEChTER, A. (1981). Chemostat studies on the hexadecane assimilation by the yeast Candida tropicalis. II. Regulation of cytochrome and enzymes. European Journal of Applied Microbiology and Biotechnology 12, $135-142$.
Pasteur, L. (1861). Influence de l'oxygene sur le développement de la levure et la fermentation alcoolique. Bulletin de la Société Chimique de Paris, June 28, 79-80.

Petrik, M., K̈̈ Ppeli, O. \& Fiechter, A. (1983). An expanded concept for the glucose effect in the yeast Saccharomyces uvarum: involvement of short- and long-term regulation. Journal of General Microbiology' $129,43-49$.

Rieger, M., Kä PPELI, O. \& Fiechter, A. (1983). The role of limited respiration in the incomplete oxidation of glucose by Saccharomyces cerevisiae. Journal of General Microbiology 129, 653-661.

RyU, D. Y. \& Mateles, R. I. (1968). Transient response of continuous cultures to changes in temperature. Biotechnology and Bioengineering 10 , 385-397.

Tempest, D. W. \& Neijssel, O. M. (1976). Microbial adaptations to low nutrient environments. In Continuous Culture 6: Applications and New Fields, pp. 283 296. Edited by H. C. R. Dean, D. C. Ellwood, C. G. T. Evans \& J. Melling. Chichester: Ellis Horwood 